\title{
In-situ micro-spectroscopy technique for chemical bonding during nucleation: A transition from soft bond to stiff bond
}

\author{
XUE DongFeng ${ }^{*} \&$ WANG HuiLin \\ State Key Laboratory of Rare Earth Resource Utilization, Changchun Institute of Applied Chemistry, Chinese Academy of Sciences, Changchun \\ 130022, China
}

Received January 16, 2020; accepted February 9, 2020; published online April 7, 2020

Citation: Xue D F, Wang H L. In-situ micro-spectroscopy technique for chemical bonding during nucleation: A transition from soft bond to stiff bond. Sci China Tech Sci, 2020, 63, https://doi.org/10.1007/s11431-020-1535-1

In-situ technique has been widely used in recent years since the advent of high-resolution spectroscopy systems, which stimulates us to probe into the time-dependent, dynamic behaviors and relevant mechanisms during a series of physical processes [1]. Especially, in-situ analysis presents a unique real-time glimpse into the fascinating physical phenomena [2], which are very important to develop the materials for subsequent applications. Generally speaking, the nucleation process involves dynamic changes in the cluster structures, as well as the formation and transformation of chemical bonds, which requires in-situ analysis urgently [3].

The idea of chemical bonding provides novel insights into the nucleation process $[4,5]$ which can be expressed as a chemical equation as follows:

$C+N_{\mathrm{s}} \rightarrow N_{1}$,

where $C$ is the cluster, $N_{\mathrm{s}}$ is the small size nucleus and $N_{\mathrm{l}}$ is the large size nucleus. This model contains two fundamental assumptions: entropy change approximation and chemical bond softening. In the first assumption, entropy change may be assumed negligible when individual small size clusters are combined into larger clusters. This means that reduction of the whole system energy comes from the released energy via forming chemical bonds when clusters become combined. This also makes it possible to use bond energy (bond enthalpy) [6] to describe free energy variation of the system. In the second assumption, ions with similar resonance status

*Corresponding author (email: dongfeng@ciac.ac.cn)
[7] often combine to form clusters, and the clustering structure is constantly changing due to chemical bonds softening. Different chemical bonding is the key to distinguish the state of the system. As shown in Figure 1, the asformed clusters via those soft chemical bonds belong to a kind of amorphous state. When the size is large enough, the soft chemical bonds transform into stiff ones, resulting in a further reduction in energy. This marks the formation of a nucleus with a crystalline structure which has the lowest energy and therefore no longer changes dynamically.

We have proposed and experimentally demonstrated the in-situ micro Raman characterization technique of chemical bonding behaviors driven by electronegativity [8]. As shown in Figure 2, in a modified setup, the emission from a single droplet can be excited in-situ under a laser scanning. The sample information about chemical bonding in aqueous system can be characterized in-situ by a series of spectra. High spatial resolution and the design of the bottom droplets make it possible to observe nucleation in real time. Raman signal increased obviously with the increase of the laser intensity $[9,10]$, and the use of droplet inversion and ultra-thin glass expand the application of high magnification short focus objectives in solution Raman testing, which further significantly improves the signal. Figure 3 is a typical Raman spectrum of hexahydrate nickel sulfate $\left(\mathrm{NiSO}_{4} \cdot 6 \mathrm{H}_{2} \mathrm{O}\right)$ aqueous solution which is divided into two regions to analyze variations of sulfate ions and water molecules [11,12]. Through rapid and continuous scanning of these two regions, 


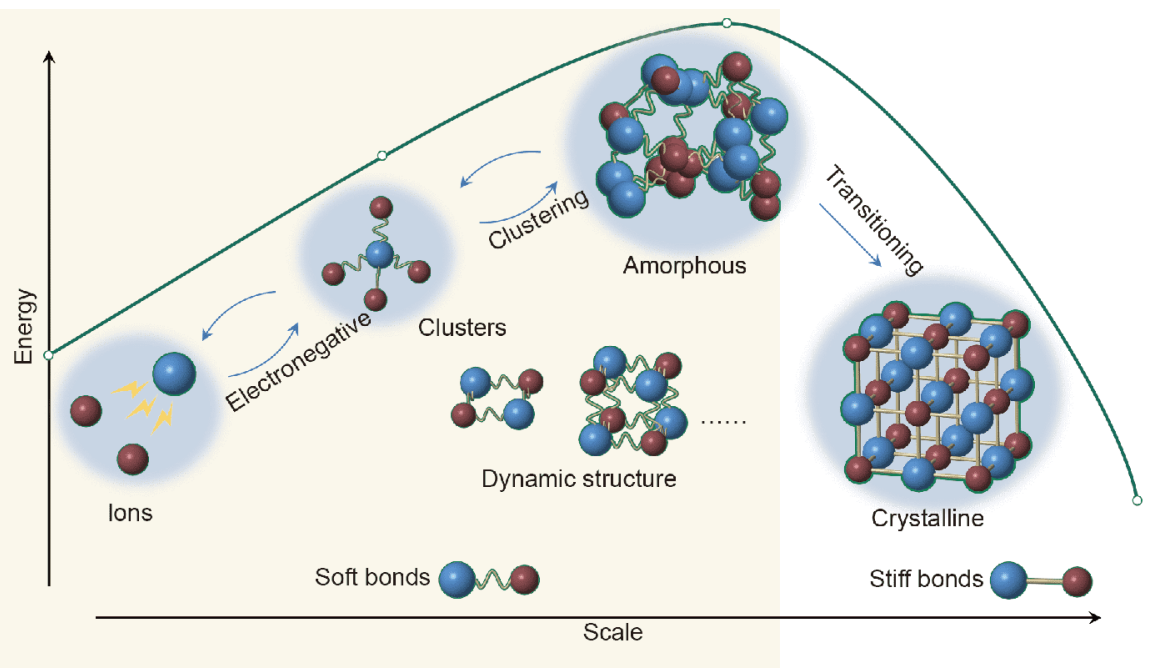

Figure 1 (Color online) Schematic drawing of chemical bonding during nucleation. Ions form clusters with dynamic structure due to electronegativity. As the supersaturation increases, the cluster size increases to a critical amorphous state. This process is the chemical bond softening which produces soft chemical bonds that are easy to recombine. Finally, the soft bond changes to stiff bond and thus becomes crystalline which the structure no longer changes dynamically.

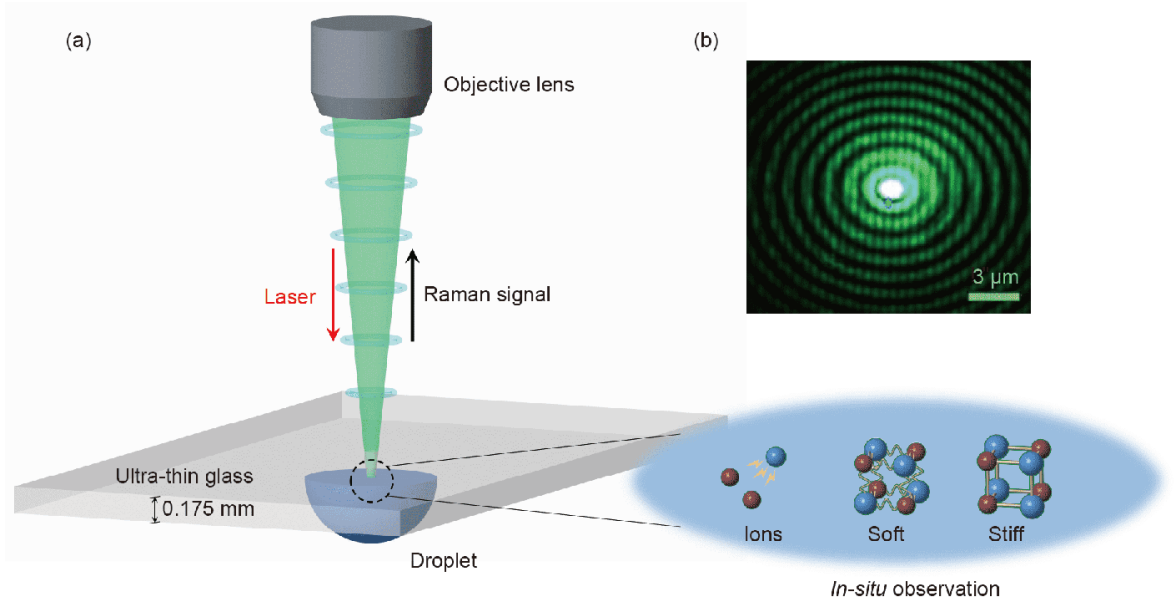

Figure 2 (Color online) (a) Schematic drawing of the in-situ Raman characterization setup; (b) enhanced signal of the laser spot focusing onto the sample droplet.

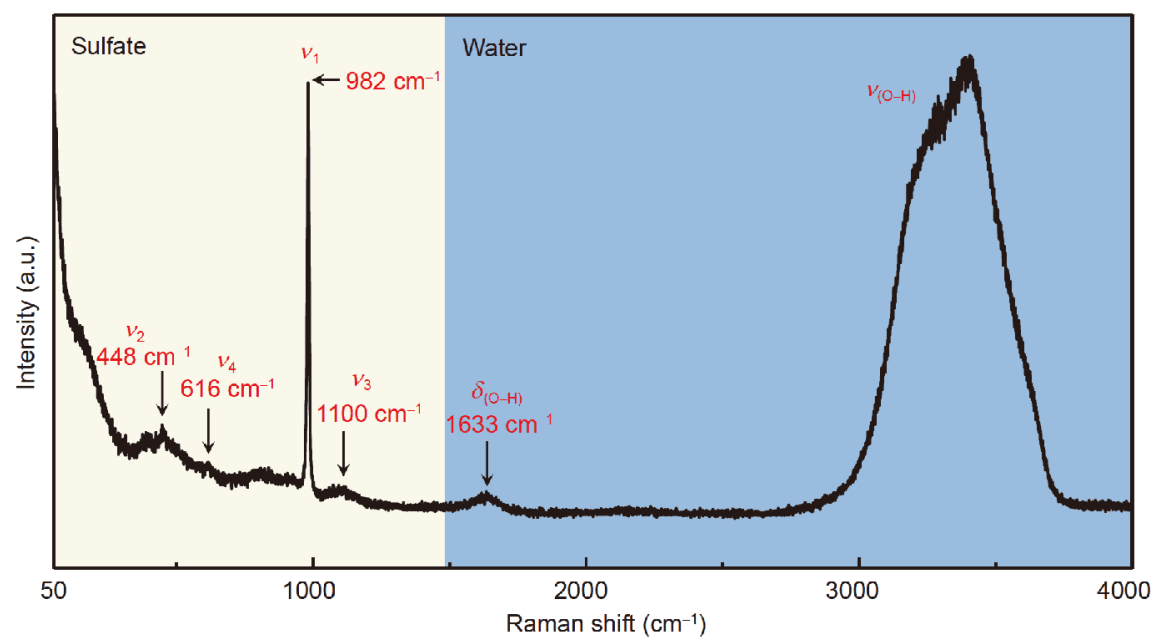

Figure 3 (Color online) Typical Raman spectrum of the $\mathrm{NiSO}_{4} \cdot 6 \mathrm{H}_{2} \mathrm{O}$ aqueous solution sample on the droplet bottom microprobe. 


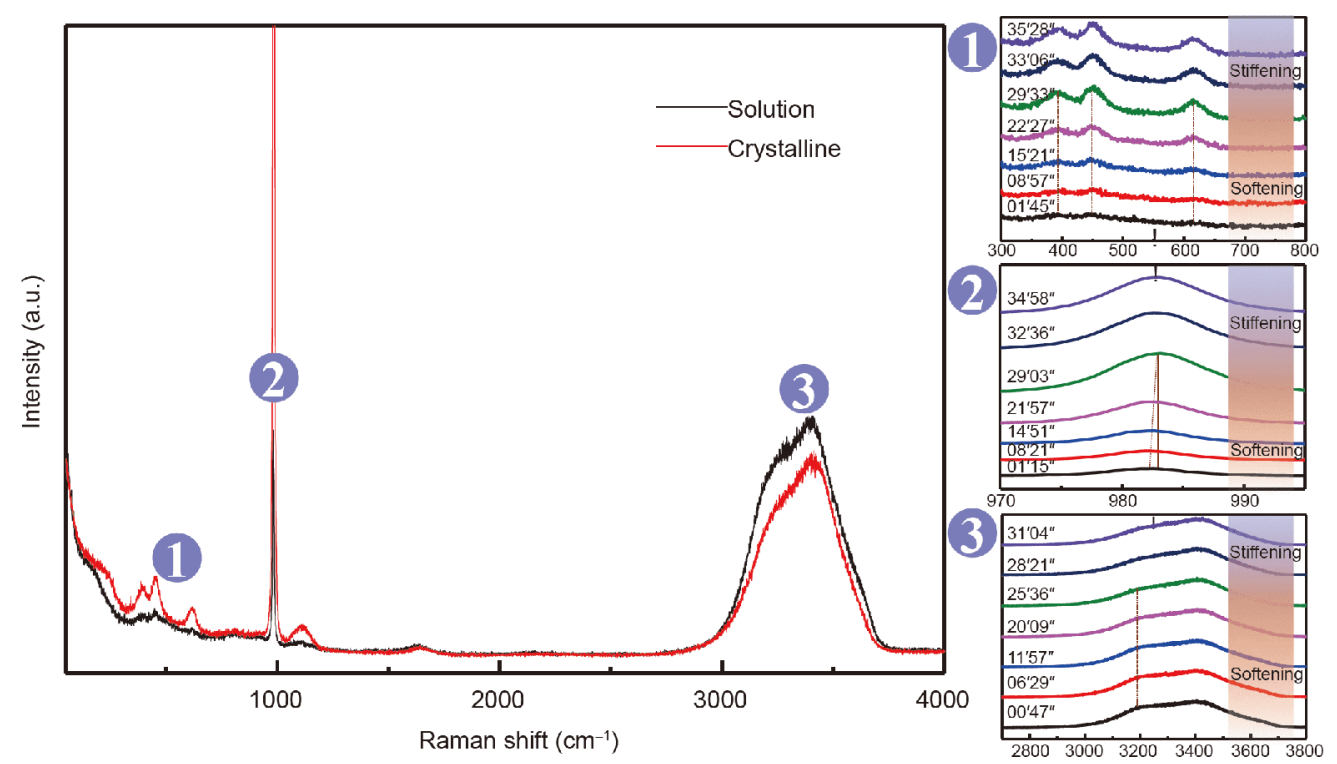

Figure 4 (Color online) Rapid and continuous Raman scanning for three regions of $\mathrm{NiSO}_{4} \cdot 6 \mathrm{H}_{2} \mathrm{O}$ aqueous solution. Due to chemical bond softening, the Raman intensity of sulfate ion vibration will gradually increase, and the intensity of water molecules will gradually decrease. After the formation of stiff bond, the intensity no longer changes.

we obtained the changes of sulfate ions and water molecules during nucleation, that is, the variation of solution structure (Figure 4). In-situ observations allow us to find the whole process of chemical bond softening and the sharp change, i.e., the transition from soft to stiff bonding. This result shows great importance of chemical bonding during nucleation process. The current strategy and method are quite general and should be applicable to the semi-quantitative research of complex crystallization processes under multiple physical field driving conditions and reaction process of multiple substances.

This work was supported by the National Natural Science Foundation of China (Grant No. 51832007). We greatly thank Professor Andreas Peter Ruediger for his huge help on our Raman spectroscopy.

1 Ma W, Lutsko J F, Rimer J D, et al. Antagonistic cooperativity between crystal growth modifiers. Nature, 2020, 577: 497-501

2 Bai G, Gao D, Liu Z, et al. Probing the critical nucleus size for ice formation with graphene oxide nanosheets. Nature, 2019, 576: 437441
3 Sun C T, Xue D F. Multisize and multiweight effects in materials science and engineering. Sci China Tech Sci, 2019, 62: 707-710

$4 \mathrm{Xu}$ D, Xue D. Chemical bond calculations of crystal growth of KDP and ADP. J Rare Earths, 2006, 24: 144-148

5 Sun C T, Xue D F. Chemical bonding in micro-pulling down process: High throughput single crystal growth. Sci China Tech Sci, 2018, 61: $1776-1778$

6 Luo Y. Comprehensive Handbook of Chemical Bond Energies. Boca Raton: Taylor \& Francis, 2007

7 Yang T, Chen J, Huang L, et al. Extremely short-lived reaction resonances in $\mathrm{Cl}+\mathrm{HD}(v=1) \rightarrow \mathrm{DCl}+\mathrm{H}$ due to chemical bond softening. Science, 2015, 347: 60-63

8 Chen X, Sun C, Wu S, et al. Molecular paradigm dependent nucleation in urea aqueous solution. Cryst Growth Des, 2017, 17: 2594-2599

9 Smith E. Modern Raman Spectroscopy: A Practical Approach. 2nd ed. Hoboken: Wiley, 2019

10 Vandenabeele P. Practical Raman Spectroscopy: An Introduction. Chichester: Wiley, 2013

11 Ghosh S, Ullah S, de Mendonça J P A, et al. Electronic properties and vibrational spectra of $\left(\mathrm{NH}_{4}\right)_{2} \mathrm{M}^{\prime \prime}\left(\mathrm{SO}_{4}\right)_{2} \cdot 6 \mathrm{H}_{2} \mathrm{O}(\mathrm{M}=\mathrm{Ni}, \mathrm{Cu})$ Tutton's salt: DFT and experimental study. SpectroChim Acta Part A-Mol Biomol Spectr, 2019, 218: 281-292

12 Mukhopadhyay A, Dubey P. Investigation of solvation of ammonium salts: A Raman spectroscopy and $a b$ initio study. J Raman Spectrosc, 2018, 49: 736-746 